\title{
Optimized Schwarz Methods for Maxwell Equations with Discontinuous Coefficients
}

\author{
Victorita Dolean ${ }^{1}$, Martin J. Gander ${ }^{2}$, Erwin Veneros ${ }^{3}$
}

\section{Introduction}

After the development of optimized Schwarz methods for the Helmholtz equation $[2,3,4,12,14]$, extensions to the more difficult case of Maxwell's equations were developed: for curl-curl formulations, see [1]. For first order formulations without conductivity, see [7], and with conductivity, see [5, 11]. For DG discretizations of Maxwell's equations, optimized Schwarz methods can be found in $[6,8,9]$, and for scattering problems with applications, see $[15,16]$.

We present here optimized Schwarz methods for Maxwell's equations in heterogeneous media with discontinuous coefficients, and show that the discontinuities need to be taken into account in the transmission conditions in order to obtain effective Schwarz methods. For diffusive problems, it was shown in [10] that jumps in the coefficients can actually lead to faster iterations, when they are taken into account correctly in the transmission conditions. We show here that for the case of Maxwell's equations with jumps along the interfaces, one can obtain a non-overlapping optimized Schwarz method that converges independently of the mesh parameter; this is not possible without coefficient jumps.

\section{Schwarz Methods for Maxwell's Equations}

The time dependent Maxwell equations are

$$
-\varepsilon \frac{\partial \mathscr{E}}{\partial t}+\nabla \times \mathscr{H}=\mathscr{J}, \quad \mu \frac{\partial \mathscr{H}}{\partial t}+\nabla \times \mathscr{E}=0,
$$

where $\mathscr{E}=\left(\mathscr{E}_{1}, \mathscr{E}_{2}, \mathscr{E}_{3}\right)^{T}$ is the electric field, $\mathscr{H}=\left(\mathscr{H}_{1}, \mathscr{H}_{2}, \mathscr{H}_{3}\right)^{T}$ is the magnetic field, $\varepsilon$ is the electric permittivity, $\mu$ is the magnetic permeability, and $\mathscr{J}$ is the applied current density. We assume that the applied current density is divergence free, $\operatorname{div} \mathscr{J}=0$.

The time dependent Maxwell equations (1) are a system of hyperbolic partial differential equations, see for example [7]. This hyperbolic system has for any interface two incoming and two outgoing characteristics. Imposing incoming characteristics is equivalent to imposing the impedance condition

\footnotetext{
Section de mathématiques, Université de Genève, 1211 Genève 4 victorita.dolean@unige.ch . martin.gander@unige.ch

erwin.veneros@unige.ch
} 


$$
\mathscr{B}_{\mathbf{n}}(\mathscr{E}, \mathscr{H}):=\frac{\mathscr{E}}{Z} \times \mathbf{n}+\mathbf{n} \times(\mathscr{H} \times \mathbf{n})=\mathbf{s} .
$$

We consider in this paper the time-harmonic Maxwell equations,

$$
-i \omega \varepsilon \mathbf{E}+\nabla \times \mathbf{H}=\mathbf{J}, \quad i \omega \mu \mathbf{H}+\nabla \times \mathbf{E}=\mathbf{0}, \quad \in \Omega,
$$

and study the heterogeneous case where the domain $\Omega$ consists of two non-overlapping subdomains $\Omega_{1}$ and $\Omega_{2}$ with interface $\Gamma$, with piecewise constant parameters $\varepsilon_{j}$ and $\mu_{j}$ in $\Omega_{j}, j=1,2$. A general Schwarz algorithm for this configuration is

$$
\left\{\begin{aligned}
-i \omega \varepsilon_{1} \mathbf{E}^{1, n}+\nabla \times \mathbf{H}^{1, n} & =\mathbf{J} & & \text { in } \Omega_{1}, \\
i \omega \mu_{1} \mathbf{H}^{1, n}+\nabla \times \mathbf{E}^{1, n} & =\mathbf{0} & & \text { in } \Omega_{1}, \\
\left(\mathscr{B}_{\mathbf{n}_{1}}+\mathscr{S}_{1} \mathscr{B}_{\mathbf{n}_{2}}\right)\left(\mathbf{E}^{1, n}, \mathbf{H}^{1, n}\right) & =\left(\mathscr{B}_{\mathbf{n}_{1}}+\mathscr{S}_{1} \mathscr{B}_{\mathbf{n}_{2}}\right)\left(\mathbf{E}^{2, n-1}, \mathbf{H}^{2, n-1}\right) & & \text { on } \Gamma, \\
-i \omega \varepsilon_{2} \mathbf{E}^{2, n}+\nabla \times \mathbf{H}^{2, n} & =\mathbf{J} & & \text { in } \Omega_{2}, \\
i \omega \mu_{2} \mathbf{H}^{2, n}+\nabla \times \mathbf{E}^{2, n} & =\mathbf{0} & & \text { in } \Omega_{2}, \\
\left(\mathscr{B}_{\mathbf{n}_{2}}+\mathscr{S}_{2} \mathscr{B}_{\mathbf{n}_{1}}\right)\left(\mathbf{E}^{2, n}, \mathbf{H}^{2, n}\right) & =\left(\mathscr{B}_{\mathbf{n}_{2}}+\mathscr{S}_{2} \mathscr{B}_{\mathbf{n}_{1}}\right)\left(\mathbf{E}^{1, n-1}, \mathbf{H}^{1, n-1}\right) & & \text { on } \Gamma,
\end{aligned}\right.
$$

where $\mathscr{S}_{j}, j=1,2$ are tangential, possibly pseudo-differential operators, and

$$
\mathscr{B}_{\mathbf{n}_{j}}\left(\mathbf{E}^{j, n}, \mathbf{H}^{j, n}\right)=\frac{\mathbf{E}^{j, n}}{Z_{j}} \times \mathbf{n}_{j}+\mathbf{n}_{j} \times\left(\mathbf{H}^{j, n} \times \mathbf{n}_{j}\right)
$$

with $Z_{j}=\sqrt{\mu_{j} / \varepsilon_{j}}, j=1,2$. Different choices of $\mathscr{S}_{j}, j=1,2$ lead to different Schwarz methods [7].

\section{The Classical Schwarz Method}

The classical Schwarz method is exchanging characteristic information at the interfaces between subdomains, which means $\mathscr{S}_{j}=0$. For the case of discontinuous coefficients and the domain $\Omega=\mathbb{R}^{3}$, with the Silver-Müller radiation condition $\lim _{r \rightarrow \infty} r(\mathbf{H} \times \mathbf{n}-\mathbf{E})=0$, and the two subdomains $\Omega_{1}=(-\infty, 0) \times \mathbb{R}^{2}, \quad \Omega_{2}=$ $(0, \infty) \times \mathbb{R}^{2}$, the classical Schwarz method does not converge in the presence of coefficient jumps:

Theorem 1. For any $\left(\mathbf{E}^{1,0}, \mathbf{H}^{1,0}\right) \in\left(L^{2}\left(\Omega_{1}\right)\right)^{6}$ and $\left(\mathbf{E}^{2,0}, \mathbf{H}^{2,0}\right) \in\left(L^{2}\left(\Omega_{2}\right)\right)^{6}$, if $\mu_{1} \varepsilon_{2} \neq$ $\mu_{2} \varepsilon_{1}$ the classical Schwarz algorithm diverges in $\left(L^{2}\left(\Omega_{1}\right)\right)^{6} \times\left(L^{2}\left(\Omega_{2}\right)\right)^{6}$.

Proof. Performing a Fourier transform in the $y z$ plane with Fourier variables $\mathbf{k}:=$ $\left(k_{y}, k_{z}\right),|\mathbf{k}|=k_{y}^{2}+k_{z}^{2}$, we obtain after a lengthy calculation similar to the one found in [7] the convergence factor

$$
\rho_{c l a}\left(\mathbf{k}, \omega_{1}, \omega_{2}, Z\right)=\max \left\{\rho_{1}\left(\mathbf{k}, \omega_{1}, \omega_{2}, Z\right), \rho_{2}\left(\mathbf{k}, \omega_{1}, \omega_{2}, Z\right)\right\}
$$

with $\omega_{1}=\omega \sqrt{\varepsilon_{1} \mu_{1}}, \omega_{2}=\omega \sqrt{\varepsilon_{2} \mu_{2}}, Z=\sqrt{\frac{\mu_{1} \varepsilon_{2}}{\mu_{2} \varepsilon_{1}}}$ and 


$$
\begin{aligned}
& \rho_{1}\left(\mathbf{k}, \omega_{1}, \omega_{2}, Z\right)=\left|\frac{\left(\sqrt{|\mathbf{k}|^{2}-\omega_{1}^{2}}-i \omega_{1} Z\right)\left(\sqrt{|\mathbf{k}|^{2}-\omega_{2}^{2}}-i \omega_{2} / Z\right)}{\left(\sqrt{|\mathbf{k}|^{2}-\omega_{1}^{2}}+i \omega_{1}\right)\left(\sqrt{|\mathbf{k}|^{2}-\omega_{2}^{2}}+i \omega_{2}\right)}\right|^{\frac{1}{2}}, \\
& \rho_{2}\left(\mathbf{k}, \omega_{1}, \omega_{2}, Z\right)=\left|\frac{\left(\sqrt{|\mathbf{k}|^{2}-\omega_{1}^{2}}-i \omega_{1} / Z\right)\left(\sqrt{|\mathbf{k}|^{2}-\omega_{2}^{2}}-i \omega_{2} Z\right)}{\left(\sqrt{|\mathbf{k}|^{2}-\omega_{1}^{2}}+i \omega_{1}\right)\left(\sqrt{|\mathbf{k}|^{2}-\omega_{2}^{2}}+i \omega_{2}\right)}\right|^{\frac{1}{2}} .
\end{aligned}
$$

The condition $\mu_{1} \varepsilon_{2} \neq \mu_{2} \varepsilon_{1}$ is equivalent to $Z \neq 1$. To show divergence, we consider 3 cases: if $\omega_{1}>\omega_{2}$, we obtain for $|\mathbf{k}|=\omega_{1}$

$\rho_{1}^{4}\left(\mathbf{k}, \omega_{1}, \omega_{2}, Z\right)=1+\frac{\left(\omega_{1}^{2}-\omega_{2}^{2}\right)\left(Z^{2}-1\right)}{\omega_{1}^{2}}, \quad \rho_{2}^{4}\left(\mathbf{k}, \omega_{1}, \omega_{2}, Z\right)=1-\frac{\left(\omega_{1}^{2}-\omega_{2}^{2}\right)\left(Z^{2}-1\right)}{\omega_{1}^{2} Z^{2}}$,

and hence if $Z>1$ we have $\rho_{2}>1$, and if $Z<1$ we have $\rho_{1}>1$. Therefore, the algorithm diverges for $\omega_{1}>\omega_{2}$. Similarly if $\omega_{1}<\omega_{2}$ we get for $|\mathbf{k}|=\omega_{2}$

$\rho_{1}^{4}\left(\mathbf{k}, \omega_{1}, \omega_{2}, Z\right)=1-\frac{\left(\omega_{2}^{2}-\omega_{1}^{2}\right)\left(Z^{2}-1\right)}{\omega_{2}^{2} Z^{2}} \quad \rho_{2}^{4}\left(\mathbf{k}, \omega_{1}, \omega_{2}, Z\right)=1+\frac{\left(\omega_{2}^{2}-\omega_{1}^{2}\right)\left(Z^{2}-1\right)}{\omega_{2}^{2}}$,

and we obtain divergence as in the first case. Finally, if $\omega_{1}=\omega_{2}$, we find

$\rho_{1}\left(\mathbf{k}, \omega_{1}, \omega_{2}, Z\right)=\rho_{2}\left(\mathbf{k}, \omega_{1}, \omega_{2}, Z\right)=\left|\frac{\left(\sqrt{|\mathbf{k}|^{2}-\omega_{1}^{2}}-i \omega_{1} Z\right)\left(\sqrt{|\mathbf{k}|^{2}-\omega_{1}^{2}}-i \omega_{1} / Z\right)}{\left(\sqrt{|\mathbf{k}|^{2}-\omega_{1}^{2}}+i \omega_{1}\right)^{2}}\right|^{1 / 2}$.

Setting now $|\mathbf{k}|=\sqrt{2} \omega_{1}$, we get after some simplifications that

$$
\rho_{1}^{4}=\frac{1}{4} \frac{\left(Z^{2}+1\right)^{2}}{Z^{2}}
$$

and $\rho_{1}^{4}>1$ is equivalent to

$$
\rho_{1}^{4}>1 \Longleftrightarrow\left(Z^{2}+1\right)^{2}>4 Z^{2} \Longleftrightarrow\left(Z^{2}-1\right)^{2}>0,
$$

which always holds, because by assumption $Z \neq 1$. So we also have divergence for the case $\omega_{1}=\omega_{2}$.

The case of continuous coefficients is analyzed in [7]. In this case, $\rho_{1}=\rho_{2}$, and $\rho_{\text {cla }}(|\mathbf{k}|)<1$ for the propagative modes, $|\mathbf{k}|<\omega_{j}, j=1,2$, and $\rho_{\text {cla }}(|\mathbf{k}|)=1$ for the evanescent modes, $|\mathbf{k}|>\omega_{j}, j=1,2$, so the algorithm is stagnating for all evanescent modes. This is also the case if $\mu_{1} \varepsilon_{2}=\mu_{2} \varepsilon_{1}$ which was excluded in Theorem 1 .

Having seen that the classical Schwarz method for Maxwell's equations in three dimensions diverges for most cases in the presence of coefficient jumps, we ana- 
lyze now the special case of the two dimensional transverse magnetic $(\mathrm{TMz})$ and transverse electric (TEz) Maxwell equations. In the TMz case, the unknowns are independent of $z$, and we have $\mathbf{E}=\left(0,0, E_{z}\right)$ and $\mathbf{H}=\left(H_{x}, H_{y}, 0\right)$. In the TEz case, $\mathbf{E}=\left(E_{x}, E_{y}, 0\right)$ and $\mathbf{H}=\left(0,0, H_{z}\right)$. Since we obtain identical results in the TMz case and the TEz case (one just has to exchange the roles of $\varepsilon$ with $\mu$ ), we only show the TMz case. Our results are again based on Fourier transforms, here in the $y$ direction with Fourier variable $k$. After a similar computation as in the proof of Theorem 1, we obtain for the classical Schwarz algorithm for the TMz case the convergence factor

$$
\rho_{c l a}\left(k, \omega_{1}, \omega_{2}, Z\right)=\left|\frac{\left(\sqrt{k^{2}-\omega_{1}^{2}}-i \omega_{1} Z\right)\left(\sqrt{k^{2}-\omega_{2}^{2}}-i \omega_{2} / Z\right)}{\left(\sqrt{k^{2}-\omega_{1}^{2}}+i \omega_{1}\right)\left(\sqrt{k^{2}-\omega_{2}^{2}}+i \omega_{2}\right)}\right|^{\frac{1}{2}} .
$$

For the TMz formulation, the classical Schwarz algorithm can be convergent in the presence of coefficient jumps:

Theorem 2. Let $\mu_{1}=\mu_{2}$. If $\varepsilon_{1}<\varepsilon_{2}$ and $\sqrt{\frac{\varepsilon_{1}}{\varepsilon_{2}}}>C_{0}$, or if $\varepsilon_{1}>\varepsilon_{2}$ and $\sqrt{\frac{\varepsilon_{2}}{\varepsilon_{1}}}>C_{0}$, $C_{0}=0.3213357548 \ldots$, then the classical Schwarz algorithm for the TMz case is convergent.

Proof. We can only give an outline of the proof: without loss of generality, we can assume that $\omega_{1}<\omega_{2}$. We then proceed in three steps: first, we show that for the evanescent modes, $k>\omega_{j}, j=1,2$ we have $\rho_{\text {cla }}<1$ if $\varepsilon_{1} \neq \varepsilon_{2}$. Second, we show that $\rho_{\text {cla }}$ at $k=0$ and $k=\omega_{1}$ is strictly less than one, and finally we show that the maximum of those two values bounds $\rho_{\text {cla }}$ for all the propagative modes $k<\omega_{j}$, $j=1,2$, where the restriction involving $C_{0}$ comes in.

Theorem 3. If $\varepsilon_{1}=\varepsilon_{2}$ and $\mu_{1} \neq \mu_{2}$, then the classical Schwarz algorithm for the TMz case is divergent.

Proof. The proof is based on divergence of the evanescent modes, as in Theorem 1.

Theorem 4. If $\mu_{1} \neq \mu_{2}, \varepsilon_{1} \neq \varepsilon_{2}$ and $Z<\frac{\sqrt{2}}{2}$, then the classical Schwarz algorithm for the TMz case is divergent.

Proof. The proof is based again on divergence of the evanescent modes.

\section{Optimized Schwarz Methods}

We have seen that the classical Schwarz method is not an effective solver for Maxwell's equations in the presence of coefficient jumps. We develop now more effective transmission conditions in order to obtain optimized Schwarz methods which take the coefficient jumps into account. Using again Fourier analysis, we can show that if $\mathscr{S}_{j}, j=1,2$ have the constant Fourier symbol 


$$
\widehat{\mathscr{S}}_{1}=-\frac{s_{2}-i \omega_{2} Z^{-1}}{s_{2}+i \omega_{2}}, \quad \widehat{\mathscr{S}}_{2}=-\frac{s_{1}-i \omega_{1} Z}{s_{1}+i \omega_{1}},
$$

then the optimized Schwarz method for the TMz case has the convergence factor

$$
\rho_{\mathrm{opt}}\left(\omega_{1}, \omega_{2}, \mu_{1}, \mu_{2}, s 1, s 2, k\right)=\left|\frac{\left(\sqrt{k^{2}-\omega_{1}^{2}}-s_{1}\right)\left(\sqrt{k^{2}-\omega_{2}^{2}}-s_{2}\right)}{\left(\sqrt{k^{2}-\omega_{1}^{2}}+s_{2} \frac{\mu_{1}}{\mu_{2}}\right)\left(\sqrt{k^{2}-\omega_{2}^{2}}+s_{1} \frac{\mu_{2}}{\mu_{1}}\right)}\right|^{\frac{1}{2}} .
$$

In order to have a more efficient algorithm, we have to choose $s_{j}, j=1,2$ such that $\rho_{\text {opt }}$ is as small as possible for all numerically relevant frequencies $k \in K:=$ $\left[k_{\min }, k_{\max }\right]$, where $k_{\min }$ is a constant depending on the geometry and $k_{\max }=c_{\max } / h$, with $c_{\max }$ a constant and $h$ denoting the mesh size, see for example [13]. We search for $s_{j}$ of the form $s_{j}=c_{j}(1+i)$ such that $c_{j}, j=1,2$ will be the solutions of the min-max problem

$$
\rho^{*}=\min _{c_{1}, c_{2} \geq 0}\left(\max _{k \in K} \rho_{\mathrm{opt}}\left(\omega_{1}, \omega_{2}, \mu_{1}, \mu_{2}, k, c_{1}(1+i), c_{2}(1+i)\right)\right) .
$$

The proofs of the following theorems are based on asymptotic analysis, and are too long and technical for this short paper; they will appear elsewhere.

Theorem 5. If $\mu_{1}<\mu_{2}$ and $\frac{\mu_{2}}{\mu_{1}}>\sqrt{2}$, and $r=\sqrt{\left|\varepsilon_{1} \mu_{1}-\varepsilon_{2} \mu_{2}\right|}$, then the asymptotic solution of the min-max problem for $h$ small is

$$
\begin{gathered}
c_{1}^{*}=\frac{1}{2} \frac{c_{\max } \mu_{1}\left(\mu_{2}+\mu_{1}-\sqrt{\mu_{2}\left(4 \mu_{1}+3 \mu_{2}\right)}\right)}{\left(\mu_{2}^{2}-2 \mu_{1}^{2}\right) h}, \quad c_{2}^{*}=\omega r, \\
\rho^{*}=\sqrt[4]{\frac{1}{2}}-\frac{2^{3 / 4}}{4} \frac{\omega\left(\mu_{2}^{2}-2 \mu_{1}^{2}\right) r}{\left(\mu_{2}+2 \mu_{1}-\sqrt{\mu_{2}\left(4 \mu_{1}+3 \mu_{2}\right)}\right)} h+\mathscr{O}\left(h^{2}\right) .
\end{gathered}
$$

If $\frac{\mu_{2}}{\mu_{1}} \leq \sqrt{2}$, then the asymptotic solution of the min-max problem is

$$
\begin{gathered}
c_{1}^{*}=\frac{1}{2 h} \frac{c_{\max }\left(\mu_{2}-\mu_{1}\right)}{\mu_{2}}, \quad c_{2}^{*}=\frac{\omega r \mu_{2}}{2} \frac{\mu_{2}+\sqrt{2 \mu_{1}^{2}-\mu_{2}^{2}}}{\mu_{1}-\mu_{2}} \\
\rho^{*}=\sqrt{\frac{\mu_{1}}{\mu_{2}}}-\sqrt{\frac{\mu_{1}}{\mu_{2}} \frac{2^{3 / 4}}{4}} \frac{\omega r\left(\mu_{2}+\sqrt{2 \mu_{1}^{2}-\mu_{2}^{2}}\right)}{\mu_{2}-\mu_{1}} h+\mathscr{O}\left(h^{2}\right) .
\end{gathered}
$$

Theorem 6. If $\mu_{1}=\mu_{2}$ and $\varepsilon_{1} \neq \varepsilon_{2}$, and $r=\sqrt{\left|\varepsilon_{1} \mu_{1}-\varepsilon_{2} \mu_{2}\right|}$, then the asymptotic solution of the min-max problem for $h$ small is given by

$$
\begin{gathered}
c_{1}^{*}=\left(\frac{c_{\max }}{h}\right)^{3 / 4}\left(\frac{\omega r}{2}\right)^{1 / 4}, \quad c_{2}^{*}=\frac{1}{4}\left(\frac{2 c_{\max }}{h}\right)^{1 / 4}(\omega r)^{3 / 4}, \\
\rho^{*}=1-\left(\frac{\omega r}{2 c_{\max }}\right)^{1 / 4} h^{1 / 4}+\mathscr{O}\left(h^{1} / 2\right) .
\end{gathered}
$$


Theorem 7. If $\mu_{1}>\mu_{2}$ and $\frac{\mu_{1}}{\mu_{2}}>\sqrt{2}$, and $r=\sqrt{\left|\varepsilon_{1} \mu_{1}-\varepsilon_{2} \mu_{2}\right|}$, then the asymptotic solution of the min-max problem for $h$ small is

$$
\begin{gathered}
c_{1}^{*}=\frac{1}{2} \frac{c_{\max } \mu_{2}\left(\mu_{1}+\mu_{2}-\sqrt{\mu_{1}\left(4 \mu_{2}+3 \mu_{1}\right)}\right)}{\left(\mu_{1}^{2}-2 \mu_{2}^{2}\right) h}, \quad c_{2}^{*}=\omega r, \\
\rho^{*}=\sqrt[4]{\frac{1}{2}}-\frac{2^{3 / 4}}{4} \frac{\omega\left(\mu_{1}^{2}-2 \mu_{2}^{2}\right) r}{\left(\mu_{1}+2 \mu_{2}-\sqrt{\mu_{1}\left(4 \mu_{2}+3 \mu_{1}\right)}\right)} h+\mathscr{O}\left(h^{2}\right),
\end{gathered}
$$

and if $\frac{\mu_{1}}{\mu_{2}} \leq \sqrt{2}$ then the asymptotic solution of the min-max problem is

$$
\begin{gathered}
c_{1}^{*}=\frac{1}{2 h} \frac{c_{\max }\left(\mu_{1}-\mu_{2}\right)}{\mu_{1}}, \quad c_{2}^{*}=\frac{\omega r \mu_{1}}{2} \frac{\mu_{1}+\sqrt{2 \mu_{2}^{2}-\mu_{1}^{2}}}{\mu_{2}-\mu_{1}} \\
\rho^{*}=\sqrt{\frac{\mu_{2}}{\mu_{1}}}-\sqrt{\frac{\mu_{2}}{\mu_{1}}} \frac{2^{3 / 4}}{4} \frac{\omega r\left(\mu_{1}+\sqrt{2 \mu_{2}^{2}-\mu_{1}^{2}}\right)}{\mu_{1}-\mu_{2}} h+\mathscr{O}\left(h^{2}\right) .
\end{gathered}
$$

Theorem 5 and Theorem 7 contain the surprising result that in the presence of jumps in the coefficients, it is possible to obtain an optimized Schwarz method for Maxwell's equations with convergence factor that does not deteriorate when the mesh parameter $h$ goes to zero, even without overlap. In the first parts of each theorem, we even see the convergence is independent of the jump in the coefficients. In the case of $\mu_{1}=\mu_{2}$ in Theorem 6 however, the convergence factor depends on $h$ and deteriorates as $h$ goes to zero, as in the case in [7] when also $\varepsilon_{1}=\varepsilon_{2}$.

\section{Numerical Results}

We now present some numerical results to illustrate the performance of the algorithms. We partition the domain $\Omega=(-1,1) \times(0,1)$ into two subdomains $\Omega_{1}=$ $(-1,0) \times(0,1)$ and $\Omega_{2}=(0,1)^{2}$. In each subdomain we select constant coefficients $\varepsilon_{j}, \mu_{j}, j=1,2$. We discretize the TMz Maxwell's equations using a finite volume method, and we impose for the test on the outer boundary the impedance boundary condition $\frac{\mathbf{E}}{Z_{j}} \times \mathbf{n}_{j}+\mathbf{n}_{j} \times\left(\mathbf{H} \times \mathbf{n}_{j}\right)=0, j=1,2$.

We first show in Figure 1 convergence histories for the classical Schwarz algorithm. On the left, we show in blue the case when $\mu_{1} \neq \mu_{2}$ and $\varepsilon_{1}=\varepsilon_{2}$, and in red the case when $\mu_{1} \neq \mu_{2}$ and $\varepsilon_{1} \neq \varepsilon_{2}$ and $Z<\frac{1}{\sqrt{2}}$, and the algorithm diverges as predicted by Theorem 3 and Theorem 4. On the right in Figure 1 we show in blue the case when $\varepsilon_{1} \neq \varepsilon_{2}$ and $\mu_{1}=\mu_{2}$, and in red the case when $\mu_{1} \neq \mu_{2}$ and $\varepsilon_{1} \neq \varepsilon_{2}$ and $Z>\frac{1}{\sqrt{2}}$, and we observe convergence, as predicted by Theorem 2 .

We next show the performance of the optimized Schwarz algorithms. We call the first parts of Theorems 5 and 7 case 1, the result in Theorem 6 case 2, and the last part of Theorems 5 and 7 case 3. In Figure 2, we show scaling experiments obtained 

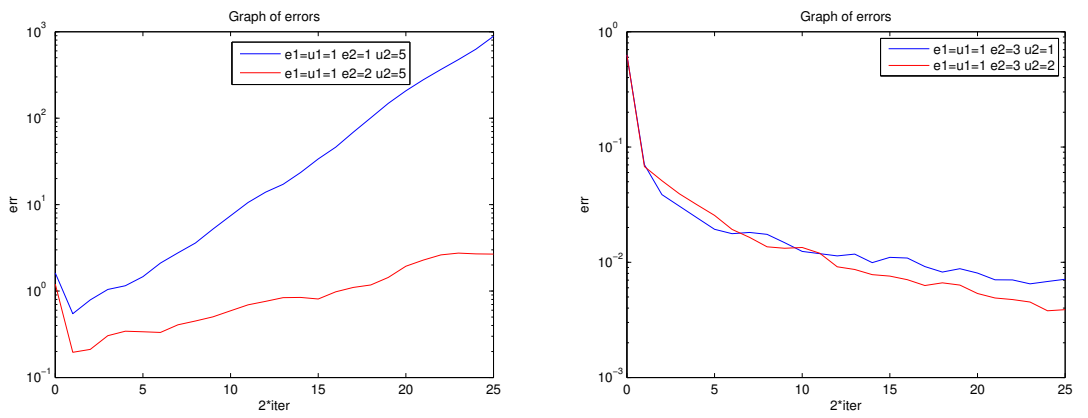

Fig. 1 Convergence histories for the classical Schwarz algorithm. On the right two cases of divergence, one where $\varepsilon$ is continuous and one where $\varepsilon$ is not continuous, and on the right two cases of convergence, one for $\mu$ continuous and one for $\mu$ not continuous

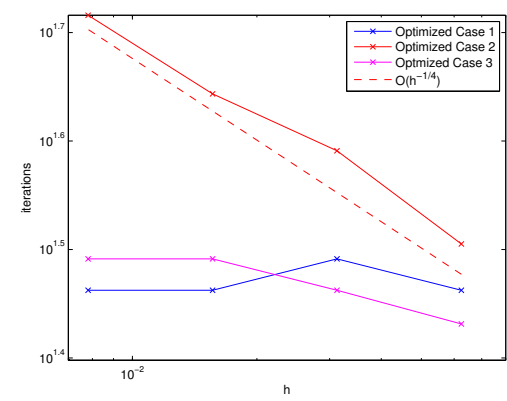

\begin{tabular}{|c|c|c|c|c|}
\hline $\mathrm{h}$ & $\frac{1}{16}$ & $\frac{1}{32}$ & $\frac{1}{64}$ & $\frac{1}{128}$ \\
\hline Optimized Case 1 & 29 & 31 & 29 & 29 \\
\hline Optimized Case 2 & 32 & 40 & 44 & 52 \\
\hline Optimized Case 3 & 27 & 29 & 31 & 31 \\
\hline
\end{tabular}

Fig. 2 Number of iterations against the mesh size $h$, to attain an error of $10^{-6}$ with the 3 cases of the optimized Schwarz algorithm

when $h$ is refined. Clearly case 1 and 3 lead to convergence independent of the mesh size, as predicted by Theorem 5 and Theorem 7, whereas the convergence in case 2 deteriorates, as predicted by Theorem 6 . We use here the parameters $\omega=2 \pi$, $\varepsilon_{1}=\mu_{1}=1$ for all the cases. For the first case we set $\varepsilon_{2}=2$ and $\mu_{2}=2$, for the second $\varepsilon_{2}=2$ and $\mu_{2}=1$ and for the third $\varepsilon_{2}=1$ and $\mu_{2}=1.4<\sqrt{2}$.

\section{Conclusions}

We proved that in the presence of jumps in the coefficients, the classical Schwarz method for Maxwell's equations in $3 \mathrm{~d}$ is not convergent, and unless $\mu_{1} \varepsilon_{2}=\mu_{2} \varepsilon_{1}$, the algorithm actually diverges. In the $2 \mathrm{~d}$ case of TMz and TEz modes, it is possible to obtain convergence for specific configurations of jumps. Optimized Schwarz methods on the other hand can take coefficient jumps into account and are always convergent, sometimes even better than without jumps. One can even get conver- 
gence independent of the mesh parameter in the non-overlapping case, something which is impossible without coefficient jumps.

\section{References}

1. Alonso-Rodriguez, A., Gerardo-Giorda, L.: New nonoverlapping domain decomposition methods for the harmonic Maxwell system. SIAM J. Sci. Comput. 28(1), 102-122 (2006)

2. Chevalier, P., Nataf, F.: An OO2 (Optimized Order 2) method for the Helmholtz and Maxwell equations. In: 10th International Conference on Domain Decomposition Methods in Science and in Engineering, pp. 400-407. AMS (1997)

3. Després, B.: Décomposition de domaine et problème de Helmholtz. C.R. Acad. Sci. Paris 1(6), 313-316 (1990)

4. Després, B., Joly, P., Roberts, J.: A domain decomposition method for the harmonic Maxwell equations. In: Iterative methods in linear algebra, pp. 475-484. North-Holland, Amsterdam (1992)

5. Dolean, V., El Bouajaji, M., Gander, M.J., Lanteri, S.: Optimized Schwarz methods for Maxwell's equations with non-zero electric conductivity. In: Domain decomposition methods in science and engineering XIX, Lect. Notes Comput. Sci. Eng., vol. 78, pp. 269-276. Springer, Heidelberg (2011). DOI 10.1007/978-3-642-11304-8_30. URL http://dx.doi.org/ $10.1007 / 978-3-642-11304-8 \quad 30$

6. Dolean, V., El Bouajaji, M., Gander, M.J., Lanteri, S., Perrussel, R.: Domain decomposition methods for electromagnetic wave propagation problems in heterogeneous media and complex domains. In: Domain decomposition methods in science and engineering XIX, Lect. Notes Comput. Sci. Eng., vol. 78, pp. 15-26. Springer, Heidelberg (2011). DOI 10.1007/978-3-642-11304-8_2. URL http://dx.doi.org/10.1007/ 978-3-642-11304-8_2

7. Dolean, V., Gerardo-Giorda, L., Gander, M.: Optimized Schwarz methods for Maxwell equations. SIAM J. Scient. Comp. 31(3), 2193-2213 (2009)

8. Dolean, V., Lanteri, S., Perrussel, R.: A domain decomposition method for solving the threedimensional time-harmonic Maxwell equations discretized by discontinuous Galerkin methods. J. Comput. Phys. 227(3), 2044-2072 (2008)

9. Dolean, V., Lanteri, S., Perrussel, R.: Optimized Schwarz algorithms for solving timeharmonic Maxwell's equations discretized by a discontinuous Galerkin method. IEEE. Trans. Magn. 44(6), 954-957 (2008)

10. Dubois, O.: Optimized Schwarz methods for the advection-diffusion equation and for problems with discontinuous coefficients. Ph.D. thesis, McGill University (2007)

11. El Bouajaji, M., Dolean, V., Gander, M.J., Lanteri, S.: Optimized Schwarz methods for the time-harmonic Maxwell equations with damping. SIAM Journal on Scientific Computing 34(4), A2048-A2071 (2012). DOI http://dx.doi.org/10.1137/110842995

12. Gander, M., Magoulès, F., Nataf, F.: Optimized Schwarz methods without overlap for the Helmholtz equation. SIAM J. Sci. Comput. 24(1), 38-60 (2002)

13. Gander, M.J.: Optimized Schwarz methods. SIAM J. Numer. Anal. 44(2), 699-731 (2006)

14. Gander, M.J., Halpern, L., Magoulès, F.: An optimized Schwarz method with two-sided robin transmission conditions for the Helmholtz equation. Int. J. for Num. Meth. in Fluids 55(2), 163-175 (2007)

15. Peng, Z., Lee, J.F.: Non-conformal domain decomposition method with second-order transmission conditions for time-harmonic electromagnetics. J. Comput. Physics 229(16), 56155629 (2010). URL http://dx.doi.org/10.1016/j.jcp.2010.03.049

16. Peng, Z., Rawat, V., Lee, J.F.: One way domain decomposition method with second order transmission conditions for solving electromagnetic wave problems. J. Comput. Physics 229(4), 1181-1197 (2010). URL http: / /dx.doi.org/10.1016/j.jcp.2009.10. 024 